\title{
Continuous on-body sensing for the COVID-19 pandemic: Gaps and opportunities
}

\author{
Hyoyoung Jeong, ${ }^{1}$ John A. Rogers, ${ }^{1,2^{*}}$ and Shuai $\mathrm{Xu}^{1,2,3}$ \\ ${ }^{1}$ Querrey Simpson Institute for Bioelectronics, Northwestern University. ${ }^{2}$ Department of Biomedical Engineering, Northwestern University McCormick School of \\ Engineering. ${ }^{3}$ Department of Dermatology, Northwestern University Feinberg School of Medicine \\ *Corresponding author. Email: jrogers@northwestern.edu
}

\section{Introduction}

As of June $20^{\text {th }}, 2020$, the Center for Disease Control's tabulations show more than 2.2 million recorded cases of COVID-19 and nearly 120,000 in deaths in the U.S. (1) Infected patients present with a wide range of symptoms, from completely asymptomatic to rapidly progressive pneumonia leading to death. Rigorous and widespread testing remains a critical component of strategies for containing this pandemic. The limited availability of molecular diagnostics constrains the use of these technologies to those who present with disease. The current gold standards rely on detection of viral RNA typically by RTPCR, but such approaches, as commonly implemented, have significant disadvantages. First, the nasopharyngeal swab is uncomfortable and may not be tolerated well by all patients, particularly children or the elderly. Second, false negative rates remain a significant concern, with some RT-PCR tests exhibiting values as high as $15 \%$ (2). Third, swab samples must be collected by trained staff to avoid false negatives or inclusive tests $(3,4)$. Fourth, samples must be transported via viral medium to centralized laboratory facilities, where delays for rural or remote areas can reach 48 hours or longer (5). Alternative tests based on antibodies offer some promise, but the appearance of antibodies (IgM and IgG) can lag weeks to months after the initial exposure (6). Furthermore, positive antibody titers may potentially only reflect prior exposure as opposed to protective immunity. Emerging evidence suggests re-infection by endemic coronaviruses is not atypical, thereby adding complications to protocols for follow-up molecular testing $(6,7)$. The net result is a continuing gap between widespread population level testing and the availability of tests, likely to persist for the foreseeable future (8-11). These circumstances motivate the development of complementary technologies for diagnosing and monitoring COVID-19 infections $(2,12,13)$.

\section{Consumer wearables to the rescue}

The demand for systems to diagnose and track COVID-19 infections non-invasively and continuously, not only in the hospital but also in-home settings motivates heightened interest in consumer-grade wearables. For instance, recent partnerships between Oura Ring (Oulu, Finland) and the University of California San Francisco (TemPREDICT Study) and West Virginia University involve digital tracking of physiological parameters in at-risk healthcare workers. AppleWatch and FitBit have similar relationships with the University of California San Diego. The underlying premise is that subtle changes in basic vital signs, not readily apparent to the individual, will offer earlier signs of infection compared to those associated with self-reported symptoms. Beyond early diagnosis, the ability to track physiological status after confirmatory molecular diagnosis may also yield insights into the process of recovery or warn of impending deterioration. While consumer-grade wearables offer the advantage of widespread availability, interoperability with widely available smartphones, and systems for remote data management, they are highly constrained in measurement modalities and accuracy. The early symptoms of COVID-19 infections are well known: fever (98\%), cough (65\%), and shortness of breath (55\%) (14-16). Patient reported symptoms elicited from mobile applications such as the loss of smell and taste offer additional prognostic utility (17). Silent hypoxemia is another sign of infection and evidence impending deterioration (18). The most popular wearables (AppleWatch, FitBit, and Oura Ring) offer continuous heart rate and physical activity measurements. They do not, however, offer pulse oximetry, body temperature, or high-fidelity measurements of respiratory rate. Furthermore, popular FitBit sensors and the Oura Ring are not FDA cleared for remote monitoring. The AppleWatch Series 4 is FDA cleared for episodic electrocardiography and irregular rhythm notification for adults above the age of 22, but with the specification that the watch is not intended to replace gold standard clinical diagnostic tools. These shortcomings may detract from the potential of these technologies to predict, monitor, and track COVID-19.

\section{On-body, skin-integrated sensor systems}

Soft electronic systems that couple intimately to the skin at locations beyond the wrist and the finger offer important advantages in assessing COVID-19 symptoms and recovery. As COVID-19 is predominately a respiratory disease, measurements of respiratory biomarkers such as cough 
frequency/intensity/sound, respiratory rate and respiratory effort, captured directly from the thorax, are likely to have high information yield. Likewise, measurements of blood oxygenation via pulse oximetry-validated to clinical grade standards-are critically important. VitalConnect (San Jose, CA) has partnered with the U.S. Department of Health and Human Service's Biomedical Advanced Research and Development (BARDA) to deploy their single-use, chest-mounted patch in nursing homes to track vulnerable elderly patients. The focus is on continuous cardiac monitoring, with basic capabilities in approximate measurements of respiratory rate. A separate, conventional pulse oximeter captures data from the finger, in an episodic mode. Efforts such as these are especially timely as long-term care facilities (LTCs) present the highest risks of infection and death from COVID-19 (19-21). Furthermore, elderly patients are often less likely to report their symptoms, and care staff in LTCs are often too overburdened to provide reliable tracking.

Our research group has also partnered with BARDA and a commercial entity, Sonica Health, to deploy advanced wearable technologies configured to directly address needs in COVID-19 monitoring, tailored specifically to the key symptoms. Here, a thin, soft sensor with a high-bandwidth accelerometer and a precision temperature sensor mounts in direct mechanical communication with the skin overlying the suprasternal notch (Fig. 1) (22). This small area of the body at the base of the neck provides an excellent interface to the intrathoracic cavity for high-fidelity recordings of respiratory activity, from cough frequency, intensity and duration, to respiratory rate and effort, to high frequency respiratory features associated with wheezing and sneezing. The same data streams include information on heart rate, heart sounds and cardiac amplitude. A thermally insulating pocket around the temperature sensor enables measurements of skin temperature, with robust correlations to core body values, largely independent of ambient conditions. The compliant mechanics of these sensors support comfortable interfaces that are compatible with the full range of natural motions of the neck. Figure 1 shows the device and some representative data collected from a patient with COVID-19. These systems, as well as a related platform developed by Sibel Health for wireless ICU-grade monitoring of complete vital signs, including blood oximetry, are currently deployed at scale on patients, physicians, nurses and respiratory rehabilitation specialists at the Shirley Ryan AbilityLab and Northwestern Memorial Hospital in Chicago (23). Initial, on-going pilot studies on $\sim 50$ subjects over the last two months reveal many interesting features of the disease and its progression, extracted from several terabytes of raw data, including those collected on patients released to the home. The overall flow of data from these devices to data analytics tools, cloud servers and physician dashboards appears in Fig. 2. Given the expected second surge of COVID-19 infections in the fall of 2020, the results of these studies, to be published in the near future, have the potential to catalyze expansion for wide-scale deployment.

\section{Data management, machine learning, and the hope for predictive algorithms}

Continuous monitoring using such technologies presents not only opportunities but also challenges in data analytics and in data management, given the exceptionally high volumes and wide diversity of health information generated by each device. Thus, these sensor systems must include scalable data backends that securely transfer, store, process and make available patient information in a HIPAA compliant manner (Fig. 2). Furthermore, the need for linking these data to other, disparate sources such as electronic health records to enhance the information content motivates the development of designs for interoperability. The challenge is that digital health data involve proprietary formats and incompatible systems, leading to fragmented silos (24). Data sharing protocols must extend across both consumer wearables and medical grade systems to combine these disparate data streams. The National Institutes of Health recently launched the National COVID Cohort Collaborative to build a centralized national data resource. While the initial focus is on increasing the wide availability of clinical data to accelerate the development of therapeutics, this framework can be leveraged as a data repository for continuous sensors and physiological outputs (25). Linking this physiological data with clinical outcomes, the effect of experimental therapeutics, and results of molecular testing will create a valuable global resource for the development of machine learning techniques to detect COVID-19 infections in the asymptomatic, to establish digital biomes of expected recovery specific to a patient's health status, and provide guidelines for workers to return to their jobs safely.

\section{Conclusion}

Continuous physiological monitoring has well-established clinical benefits across a wide range of medical use cases when deployed in non-critical care settings (26). For COVID19 , the extension of continuous monitoring from the hospital to the home offers hope. Ongoing clinical studies will demonstrate the extent of this benefit with technologies that measure parameters directly relevant to the infection, with clinical-grade accuracy. A focus on data interoperability and sharing will accelerate the development of predictive algorithms that are generalizable across different populations. In parallel with global efforts to develop effective vaccines and drugs to prevent and treat COVID-19, continuous skin-integrated sensors, positioned at optimal locations of the anatomy, will address the ongoing and critical need for objective, continuous, and sensitive tools to detect COVID-19 symptoms 
early in the general population, to triage limited molecular testing, to recognize the need for an escalation of care, and to track recovery curves.

This study was conducted under guidelines approved by the Northwestern University Institutional Review Board under protocol STU00212522.

The work was supported by the Biomedical Advanced Research and Development's DRIVe Early Notification to Act, Control, and Treat Program (75A50119C00043).

\section{REFERENCES AND NOTES}

1. Cases in the US, Coronavirus Disease 2019. CDC. Accessed June 20th, 2020. https://www.cdc.gov/coronavirus/2019-ncov/cases-updates/cases-inus.html.

2. J. D. Whitman et al., Test performance evaluation of SARS-CoV-2 serological assays. https://www.medrxiv.org/content/10.1101/2020.04.25.20074856v2.

3. World Health Organization. Laboratory testing strategy recommendations for COVID-19. 22 Mar 2020

4. W. Wang, Y. Xu, R. Gao, R. Lu, K. Han, G. Wu, W. Tan, Detection of SARS-CoV-2 in Different Types of Clinical Specimens. JAMA (2020). doi:10.1001/jama.2020.3786 Medline

5. N. J. Beeching, T. E. Fletcher, M. B. J. Beadsworth, Covid-19: Testing times. BMJ 369, m1403 (2020). doi:10.1136/bmi.m1403 Medline

6. J. Zhao, Q. Yuan, H. Wang, W. Liu, X. Liao, Y. Su, X. Wang, J. Yuan, T. Li, J. Li, S. Qian, C. Hong, F. Wang, Y. Liu, Z. Wang, Q. He, Z. Li, B. He, T. Zhang, Y. Fu, S. Ge, L. Liu, J. Zhang, N. Xia, Z. Zhang, Antibody responses to SARS-CoV-2 in patients of novel coronavirus disease 2019. Clin. Infect. Dis. ciaa344 (2020). doi:10.1093/cid/ciaa344 Medline

7. Marta Galanti and Jeffrey Shaman, Direct observation of repeated infections with endemic coronaviruses. 2020.2 Pre-print: http://www.columbia.edu/ jls106/galanti shaman ms supp.pdf.

8. Yelin I et al. Evaluation of COVID-19 RT-qPCR test in multi-sample pools. medXriv. https://www.medrxiv.org/content/10.1101/2020.03.26.20039438v1.

9. C. Knaus, B. Doherty, Global shortage of Covid-19 test kits hits Australia as other nations limit exports. The Guardian. 13 Mar 2020. Available: https://www.theguardian.com/australia-news/2020/mar/13/global-shortageof-covid-19-test-kits-hits-australia-as-other-nations-limit-exports. Accessed 16 Mar 2020.

10. L. Ramsey, A. Woodward, The US is struggling to test more people for the coronavirus. Now it's facing a shortage of the materials used to run those tests. Business Insider. $12 \quad$ Mar $2020 . \quad$ Available: https://www.businessinsider.com/coronavirus-testing-may-be-limited-byshortage-of-materials-2020-3 Accessed 16 Mar 2020.

11. S. Esfandiari, Wuhan, China, faces such a shortage of coronavirus test-kits that people say getting one is like "winning the lottery." Business Insider. 24 Jan 2020. Available: https://www.businessinsider.com/wuhan-coronavirus-chinashortage-test-kits-lottery-2020-1. Accessed 16 Mar 2020.

12. Erdman S. Public, private health labs may never be able to meet demand for coronavirus testing over supply chain shortages. April 29th, 2020. CNN Health.

13. Sweet $L$ and Schuba T. COVID-19 test shortage forces Chicago area clinics to halt curbside testing. May 14th, 2020. Chicago Sun Times.

14. E. J. Chow et al., Symptom Screening at Illness Onset of Health Care Personnel With SARS-CoV-2 Infection in King County, Washington. JAMA, (2020).

15. T. Greenhalgh, G. C. H. Koh, J. Car, Covid-19: A remote assessment in primary care. BMJ 368, m1182 (2020). doi:10.1136/bmi.m1182 Medline

16. W. J. Guan, Z. Y. Ni, Y. Hu, W. H. Liang, C. Q. Ou, J. X. He, L. Liu, H. Shan, C. L. Lei, D. S. C. Hui, B. Du, L. J. Li, G. Zeng, K.-Y. Yuen, R. C. Chen, C. L. Tang, T. Wang, P. Y. Chen, J. Xiang, S. Y. Li, J. L. Wang, Z. J. Liang, Y. X. Peng, L. Wei, Y. Liu, Y. H. Hu, P. Peng, J. M. Wang, J. Y. Liu, Z. Chen, G. Li, Z. J. Zheng, S. Q. Qiu, J. Luo, C. J. Ye, S. Y. Zhu, N. S. Zhong; China Medical Treatment Expert Group for Covid-19,
Clinical Characteristics of Coronavirus Disease 2019 in China. N. Engl. J. Med. 382, 1708-1720 (2020). doi:10.1056/NEJMoa2002032 Medline

17. C. Menni, A. M. Valdes, M. B. Freidin, C. H. Sudre, L. H. Nguyen, D. A. Drew, S. Ganesh, T. Varsavsky, M. J. Cardoso, J. S. El-Sayed Moustafa, A. Visconti, P. Hysi, R. C. E. Bowyer, M. Mangino, M. Falchi, J. Wolf, S. Ourselin, A. T. Chan, C. J. Steves, T. D. Spector, Real-time tracking of self-reported symptoms to predict potential COVID-19. Nat. Med. (2020). doi:10.1038/s41591-020-0916-2 Medline

18. M. J. Tobin, F. Laghi, A. Jubran, Why COVID-19 Silent Hypoxemia is Baffling to Physicians. Am. J. Respir. Crit. Care Med. rccm.202006-2157CP (2020). doi:10.1164/rccm.202006-2157CP Medline

19. A. Morris, (Northwestern University., Chicago, IL., 2020).

20. G. Onder, G. Rezza, S. Brusaferro, Case-Fatality Rate and Characteristics of Patients Dying in Relation to COVID-19 in Italy. JAMA (2020). doi:10.1001/iama.2020.4683 Medline

21. Karen Yourish et al, One-Third of All U.S. Coronavirus Deaths Are Nursing Home Residents or Workers. New York Times. 5/9/2020.

22. K. Lee, X. Ni, J. Y. Lee, H. Arafa, D. J. Pe, S. Xu, R. Avila, M. Irie, J. H. Lee, R. L. Easterlin, D. H. Kim, H. U. Chung, O. O. Olabisi, S. Getaneh, E. Chung, M. Hill, J. Bell, H. Jang, C. Liu, J. B. Park, J. Kim, S. B. Kim, S. Mehta, M. Pharr, A. Tzavelis, J. T. Reeder, I. Huang, Y. Deng, Z. Xie, C. R. Davies, Y. Huang, J. A. Rogers, Mechanoacoustic sensing of physiological processes and body motions via a soft wireless device placed at the suprasternal notch. Nat. Biomed. Eng. 4, 148-158 (2020). doi:10.1038/s41551-019-0480-6 Medline

23. H. U. Chung, A. Y. Rwei, A. Hourlier-Fargette, S. Xu, K. Lee, E. C. Dunne, Z. Xie, C. Liu, A. Carlini, D. H. Kim, D. Ryu, E. Kulikova, J. Cao, I. C. Odland, K. B. Fields, B. Hopkins, A. Banks, C. Ogle, D. Grande, J. B. Park, J. Kim, M. Irie, H. Jang, J. Lee, Y. Park, J. Kim, H. H. Jo, H. Hahm, R. Avila, Y. Xu, M. Namkoong, J. W. Kwak, E. Suen, M. A. Paulus, R. J. Kim, B. V. Parsons, K. A. Human, S. S. Kim, M. Patel, W. Reuther, H. S. Kim, S. H. Lee, J. D. Leedle, Y. Yun, S. Rigali, T. Son, I. Jung, H. Arafa, V. R. Soundararajan, A. Ollech, A. Shukla, A. Bradley, M. Schau, C. M. Rand, L. E. Marsillio, Z. L. Harris, Y. Huang, A. Hamvas, A. S. Paller, D. E. Weese-Mayer, J. Y. Lee, J. A. Rogers, Skin-interfaced biosensors for advanced wireless physiological monitoring in neonatal and pediatric intensive-care units. Nat. Med. 26, 418-429 (2020). doi:10.1038/s41591-020-0792-9 Medline

24. M. Lehne, J. Sass, A. Essenwanger, J. Schepers, S. Thun, Why digital medicine depends on interoperability. NPJ Digit. Med. 2, 79 (2019). doi:10.1038/s41746019-0158-1 Medline

25. About the National COVID Cohort Collaborative, June 6th, 2020. Accessed: 6/21/2020. https://ncats.nih.gov/n3c/about.

26. C. L. Downey, S. Chapman, R. Randell, J. M. Brown, D. G. Jayne, The impact of continuous versus intermittent vital signs monitoring in hospitals: A systematic review and narrative synthesis. Int. J. Nurs. Stud. 84, 19-27 (2018). doi:10.1016/i.jinurstu.2018.04.013 Medline

Submitted 23 June 2020

Accepted 24 June 2020

Published First Release 1 July 2020

10.1126/sciadv.abd4794 

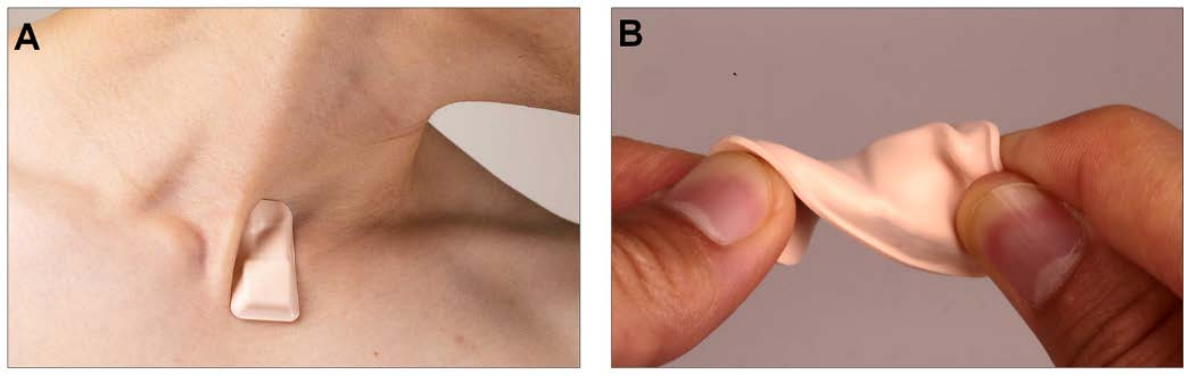

C
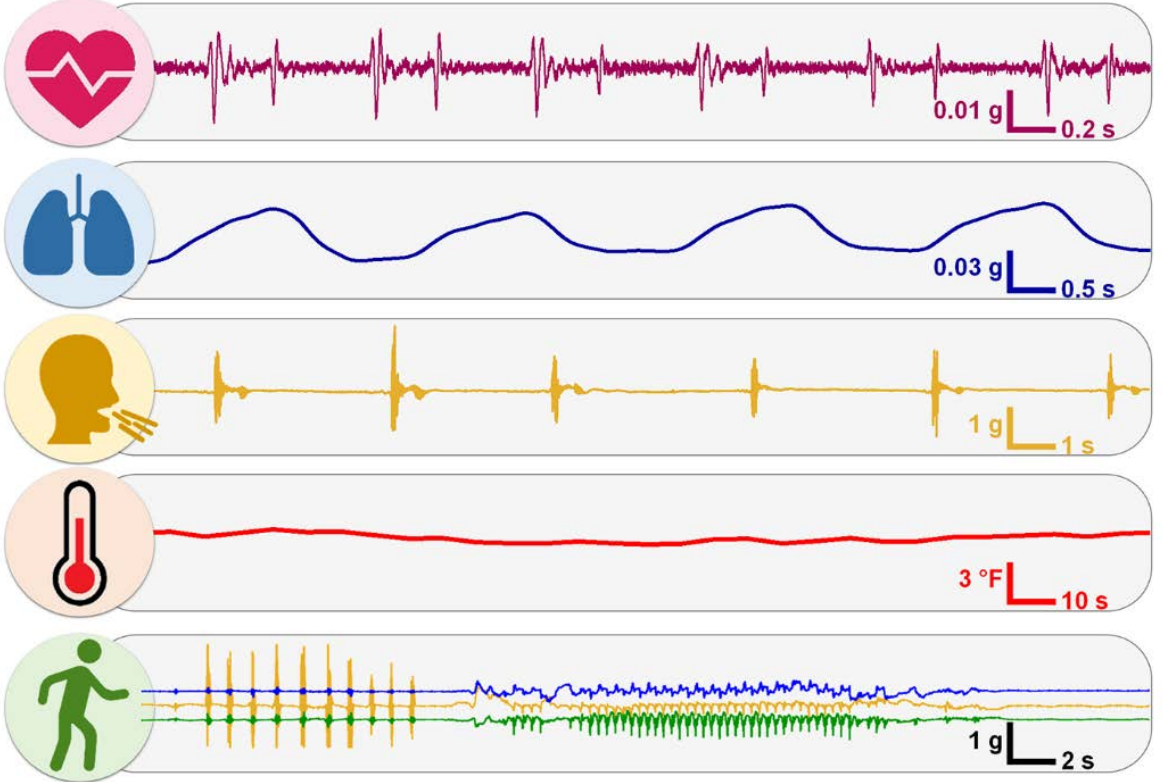

Fig. 1. Wireless, soft, skin-interfaced sensor platform designed for mounting on the suprasternal notch. (A) Image to highlight the ability of the device to conform to natural movements of the neck, without mechanical constraint, as the basis for a comfortable interface for measurements of respiratory activity and core vital signs. (B) Image of the device under severe twisting deformation. (C) Continuous wearable monitoring: cardiac- and respiratory-related signals, coughing, body temperature, and patterns of activity collected from a COVID-19 patient. 
Mobile Application

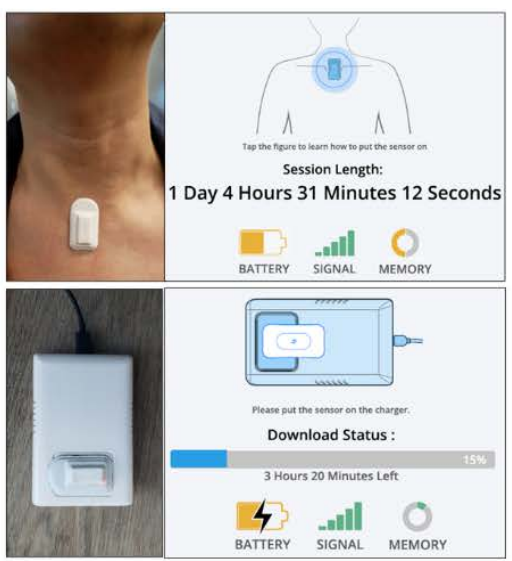

Cloud Platform

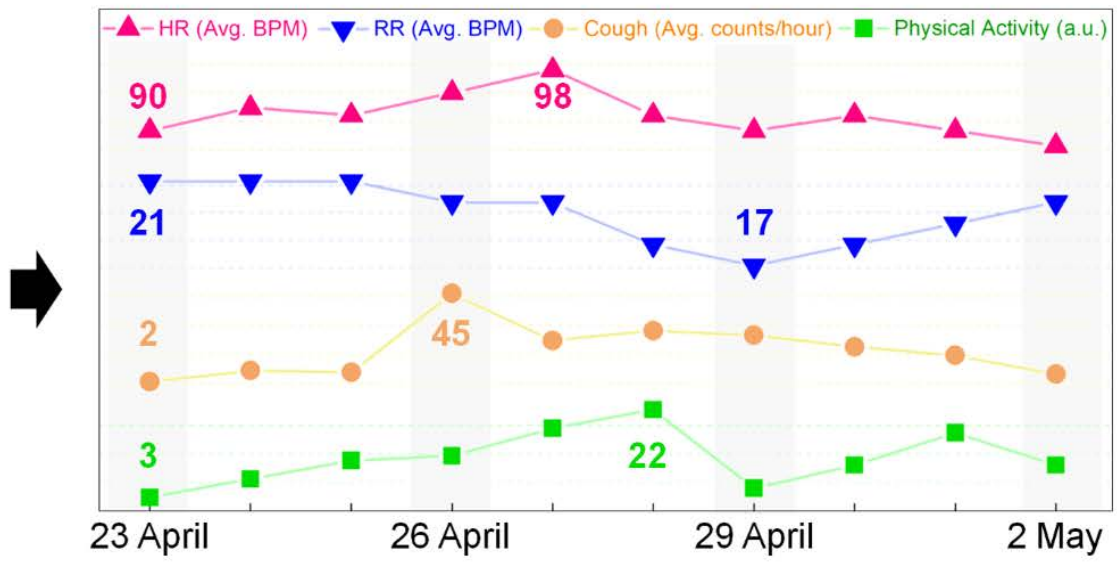

Fig. 2. Automated data management system for individual data acquisition, secure data upload, and machine learning algorithms for assessing COVID-19 symptoms and recovery. Representative data collected from a COVID-19 patient. 